\title{
Active mixing of immobilised enzymatic system in microfluidic chip
}

\author{
Kirill A. Lukyanenko ${ }^{凶}$, Kirill I. Belousov², Ivan A. Denisov ${ }^{1}$, Anton S. Yakimov ${ }^{1}$, Elena N. Esimbekova ${ }^{1,3}$, \\ Anton S. Bukatin ${ }^{1}$, Anatoly A. Evstrapov ${ }^{4}$, Peter I. Belobrov ${ }^{1,3}$ \\ ${ }^{1}$ Siberian Federal University, Krasnoyarsk 660041, Russia \\ ${ }^{2}$ ITMO University, Saint Petersburg 197101, Russia \\ ${ }^{3}$ Institute of Biophysics SB RAS, Krasnoyarsk 660036, Russia \\ ${ }^{4}$ Institute for Analytical Instrumentation, Saint Petersburg 198095, Russia \\ 凶E-mail: kirill.lukyanenko@gmail.com
}

Published in Micro \& Nano Letters; Received on 7th November 2016; Revised on 3rd February 2017; Accepted on 13th February 2017

Parameters for sample introduction, dried reagents dissolution and mixing with sample for bienzyme system NAD(H):FMN-oxidoreductase and luciferase immobilised in microfluidic chip were successfully determined. Numerical simulations of reaction chamber geometry, flavin mononucleotide (FMN) escape from starch gel and mixing options were conducted to achieve higher sensitivity of bioluminescent reaction. Results of numerical simulations were verified experimentally. The active mixer for dried reagents was made from an electromechanical speaker's membrane which was connected to the input of the chip. Such a mixer provided better efficiency than a passive mixing, and it is simple enough for use in point-of-care devices with any systems based on immobilised enzymes in chips.

1. Introduction: One of the upcoming trends in analytical instrumentation is switching routine analytical assays into miniaturised and automated point-of-care (POC) devices [1, 2]. The essential components of such devices are disposable chips which are inexpensive and easy to produce $[3,4]$. These smart biochips accommodate all the assay components i.e. a sample preparation chamber, a reagents mixing platform and provision for simultaneous signal detection with minimum periphery equipment. Disposable chips have a huge potential in many fields of medicine and ecology through their customisation.

In the present research, we describe disposable microfluidic chips for environmental monitoring using bacterial luciferase-based bioassay. The biological module described herein is based on a bienzyme bioluminescent system [5]. The interaction of contaminants in water samples with enzymes of bioluminescent bacteria leads to parametric changes in the bioluminescent system catalysed by NAD(P)H:FMN-oxidoreductase and luciferase [6]

$$
\begin{gathered}
\mathrm{FMNH}_{2}+\mathrm{RCHO}+\mathrm{O}_{2} \stackrel{\text { Luciferase }}{\longrightarrow} \mathrm{RCOOH}+\mathrm{H}_{2} \mathrm{O}+h v, \quad \text { (1) } \\
\mathrm{NADH}+\mathrm{FMN}+\mathrm{H}^{+} \stackrel{\mathrm{NAD}(\mathrm{P}) \mathrm{H}: \mathrm{FMN}-\text { oxidoreductase }}{\longrightarrow} \mathrm{FMNH}_{2}+\mathrm{NAD}^{+} .
\end{gathered}
$$

These changes are expressed in the reduction of maximum light emission intensity as compared with the control sample. Thus, it is possible to obtain an integral assessment of the analyte toxicity.

The biological module was immobilised in a starch matrix and subsequently dried and sealed in the designed microfluidic chip. The immobilised components of the reaction chamber (Fig. 1) must mix uniformly during aqueous sample introduction (environmental contaminants) to respond precisely. These pre-requisites required special arrangements to be made to promote efficient mixing since reagents (i) needed to exit from the gel followed by (ii) their uniform distribution across the reaction chamber to avoid erroneous results.

The requirement for compactness and placement of all analytical steps into a single chip makes the reagent mixing process complicated due to low Reynolds numbers [7]. Today there are many passive and active mixers available for different types of chemical reactions [8]. Most of commercially successful POC devices use passive mixing [9].
However, different passive mixers are based on curved microchannels and colliding substreams [10] which are not effective enough when dealing with dried reagents. Among many types of active mixers [8], only acoustic mixers have the potential to provide necessary mixing efficiency without affecting enzyme activity.

The aim of the current research was to determine the parameters of sample introduction, efficient dissolution of dried reagents and their subsequent uniform mixing with the assay sample in the microfluidic chip to make enzymatic bioassays applicable in environmental monitoring.

Air bubbles formation is a key factor that contributes to the subsequent qualitative mixing. It is an unwanted phenomenon which leads to the decrease of the measurement validity. Usually this occurs during the introduction of the sample into the chip. The lower the rate of the sample entry, the less is the likelihood of bubbles formation. At the same time, the sample introduction rate must be high enough to avoid any premature dissolution and passive mixing of immobilised substrates of the bioluminescent reaction. Hence it was necessary (i) to determine the optimal shape of the reaction chamber and to calculate the optimal flow velocity for sample entry without bubble formation using numerical simulation.

Due to the passive drying of flavin mononucleotide (FMN) in a starch gel its total amount participating in the reaction will depend on the characteristics of FMN escape from the gel. It is necessary (ii) to create an adequate model of FMN escape to predict this amount and determine suitable time for reagents dissolution.

Enhanced signal response and reproducibility of bioluminescent reaction requires uniform distribution of FMN concentration in the reaction chamber during the analysis. The time resolved spectroscopy method could be used to assess the FMN concentration since FMN has a colour. Finding the optimum mixing algorithm required (iii) selecting the right amplitude and frequency of liquid oscillations in the chip, which would provide the lowest coefficient of variation $(\mathrm{CV})$ of FMN concentration throughout the reaction chamber.

\section{Experimental}

2.1. Microfluidic chips: The body of the chip was made using poly (methyl methacrylate) (PMMA) of industrial quality by micromilling method with the $\mathrm{CNC}$ milling machine Modela 


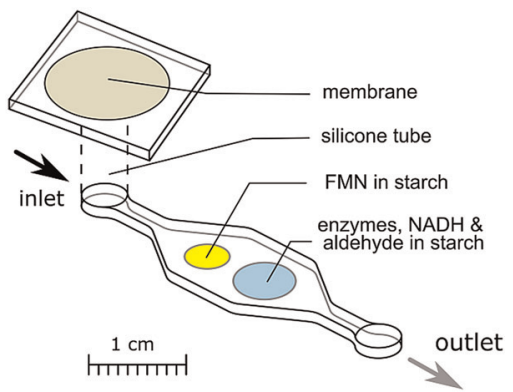

Fig. 1 Schematic representation of the microfluidic chip with connected mixer

MDX-20 (Roland, Japan). The depth of the channels was $0.5 \mathrm{~mm}$. The chips were sealed at room temperature using 1,2-dichloroethane and controlled pressure. The design of the chip with biological components is shown in Fig. 1.

2.2. Biological module: The biological module inhabited two dried starch gel droplets. The first $10 \mu \mathrm{l}$ droplet contained coupled enzymatic system: bacterial luciferase (Photobacterium leiognathi) and $\mathrm{NAD}(\mathrm{P}) \mathrm{H}: \mathrm{FMN}-$ oxidoreductase (Vibrio fischeri) in addition to reduced nicotinamide adenine dinucleotide (NADH) and tetradecanal. The second $5 \mu$ droplet contained FMN to initiate the bioluminescence reaction. Both droplets were dried on a gelatin scaffold on the surface of the reaction chamber.

2.3. Numerical simulations: The calculations of flow rate and the diffusion of FMN in microchannels were conducted with COMSOL Multiphysics (COMSOL, Sweden) using the finite element method. The system of Navier-Stokes equations was used to simulate a liquid velocity profile. The displacement of the border between the two phases during the chip filling was calculated by the level set method. The calculation of convective and diffusive transport was performed with Fick's second law with the added convective term. The quality of mixing was determined by calculating the CV. We used 2D-axisymmetric and two-dimensional (2D) models for simulations.

2.4. Concentration measurement: Uniform distribution of the reagents in the reaction chamber of the microfluidic chip was assessed through time-resolved spectroscopy by HSV palette analysis using AxioCam ICc 5 and Axio Scope A1 microscope (Carl Zeiss, Germany). The image of the reaction chamber was acquired with the camera and then the saturation level of HSV palette was analysed with proprietary software. The software was written with BlackBox Component Builder (Oberon microsystems Inc., Switzerland).

2.5. Mixing: The active mixer was made from a speaker's electromechanical membrane, which was connected to the input

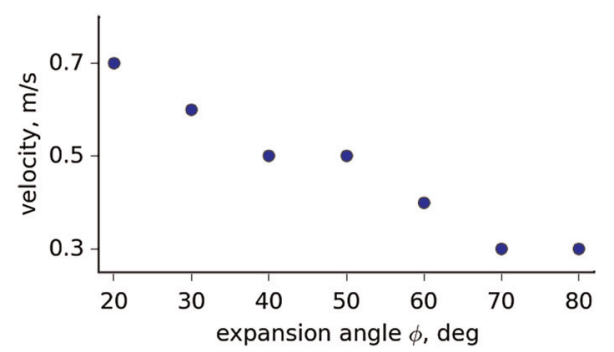

Fig. 2 Minimum velocity for different expansion angles $\varphi$ when bubble formation begins

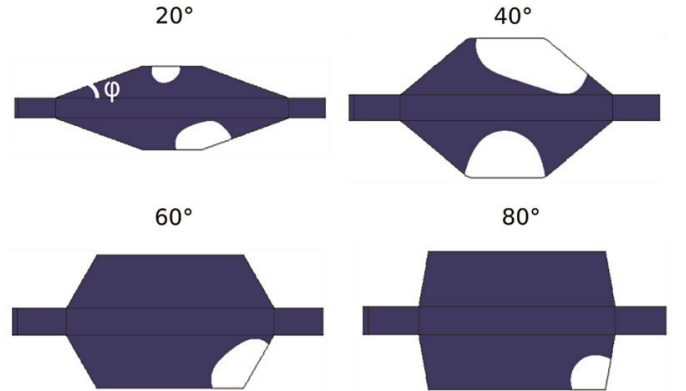

Fig. 3 Visualisation of the expansion angle $\varphi$ and examples of bubbles (white) that formed during the filling of the reaction chamber with water (blue) during simulation

of the inlet channel of the chip. The membrane was $14 \mathrm{~mm}$ in diameter and the resistance was $33 \mathrm{Ohm}$. The signal pattern for membrane movement was generated by a laboratory self-made device. It consisted of the LPC2103 MCU (NXP, Netherlands), several optrons and transistors, which controlled the $\mathrm{H}$ bridge, allowing voltage to be applied across the membrane in either direction. Signals were programmed and sent to this amplifier using the software made with BlackBox Component Builder (Oberon microsystems Inc., Switzerland). Membrane oscillations with predefined patterns created the acoustic wave, which caused fluid movement in the chip.

\section{Results and discussion}

3.1. Reaction chamber design: While filling the chip's reaction chamber with liquid during the introduction of the sample, there is a risk of air bubbles formation. These bubbles can affect the bioluminescent reaction and decrease measurement validity. One way to solve this problem is to increase the hydrophilicity of the channels. However, channel treatment with UV radiation [11, 12], plasma $[13,14]$ or other methods may damage the biological materials.

Due to immobilisation of biological components in the form of two droplets of dried gel, the diamond shape of the reaction chamber was chosen. The formation of bubbles can be minimised by optimising the geometry of the diamond-shaped reaction chamber, namely the expansion angle $\varphi$ under which it widens. Using numerical simulation for optimisation, we considered the threshold fluid flow velocity in the reaction chamber that triggers bubbles formation. At optimal flow speed, the probability of bubbles formation was minimised.

Water filled the empty reaction chamber during the simulation process. Different reaction chambers with various expansion angles were considered. All variants ranged with $\varphi$ (Fig. 3): from 20 to $80^{\circ}$. With every geometry, the reaction chamber remained relatively compact, not $>15 \mathrm{~mm}$ in length and $6 \mathrm{~mm}$ in width. The square area remained approximately equal to $40 \mathrm{~cm}^{2}$ in all cases. The reaction chamber was initially filled with air.

The water filled the reaction chamber at constant velocity. Boundary condition for walls was wetted wall. The wetting angle for PMMA used in this work was $70 \pm 5^{\circ}$.

The minimum flow velocity at which bubble formation begins at different $\varphi$ angles was studied. As shown in Fig. 2, the results of the numerical simulation indicate that minimum flow velocity at which bubble formation begins gradually reduces from $0.7 \mathrm{~m} / \mathrm{s}$ for $20^{\circ}$ to $0.3 \mathrm{~m} / \mathrm{s}$ at $80^{\circ}$. The examples of main types of bubbles are shown in Fig. 3.

On the basis of these results, in further experiments, we used $30^{\circ}$ expansion angle (Fig. 1) in the reaction chamber. This angle was chosen due to the optimum dimensions of the reaction chamber and high flow threshold velocity of $0.6 \mathrm{~m} / \mathrm{s}$ at which the bubble 


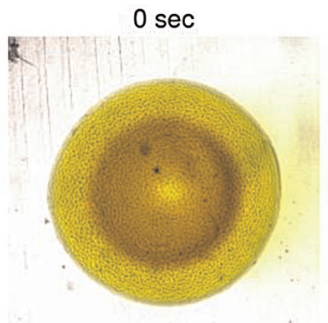

$20 \mathrm{sec}$

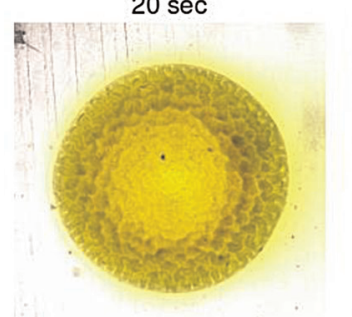

$10 \mathrm{sec}$

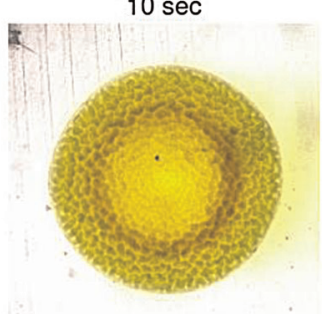

$30 \mathrm{sec}$

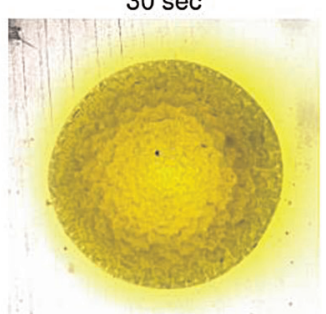

Fig. 4 FMN diffusion from the starch droplet in the microfluidic chip after water sample injection

formation may begin. This high velocity ensures the lowest probability of bubble formation during the introduction of the sample.

3.2. FMN escape from the starch gel: The processes involving FMN and enzymatic escape from the starch gel has significant influence on the quality of mixing. It is necessary to determine the time at which most of the FMN escapes the starch gel to achieve uniform FMN distribution in the reaction chamber after mixing.

The simulation results were compared with the experimental data of FMN diffusion (Fig. 4) that were acquired using the timeresolved spectroscopy method. The experimental rates of concentration change $\mathrm{d} c / \mathrm{d} t$ were obtained, the average interval being $0.4 \mathrm{~s}$. The data were noisy due to the small size of the gel droplet $(5 \mu \mathrm{l})$ and the error in the measurement. Nevertheless, successful smoothing was performed using the fast Fourier transform filter with cut-off frequency equal to 0.08 (Fig. 5, green line). In Figs. 5 and 6 the presented concentration value per unit area relates to the $2 \mathrm{D}$ nature of experimental images. It should be noted that the parameter of FMN distribution in gel and the dynamics of gel swelling remain unknown.

The process of FMN escape involves two steps: dissolution of FMN crystals in the liquid and diffusion of FMN from the gel. The simulation showed that dissolution led to a continuous decrease of the escape velocity while the experiment indicated the increase of escape velocity at the beginning. Hence the diffusion spread has a priority. In the following simulation only diffusion was considered.

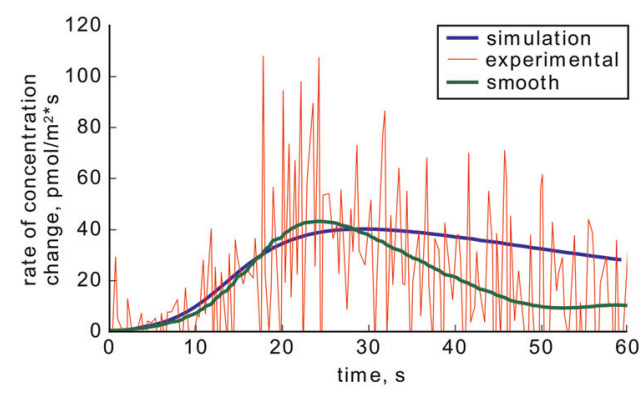

Fig. 5 Rate of concentration change dc/dt of numerical simulation and experimental data $80 \mu \mathrm{l}$ from the gel boundary. Blue - simulation, red - experimental data, green - smoothed data

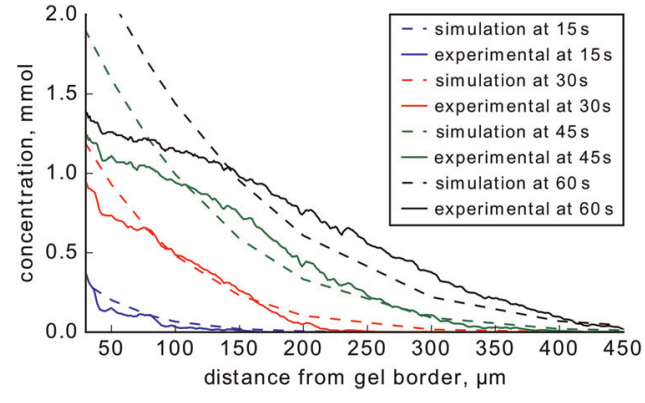

Fig. 6 Concentration distribution profile at various times. Solid lines experimental data, dashed lines - simulation data

The fitting of the simulation results was carried out based on the value of the rate of the concentration change at a distance of $80 \mu \mathrm{m}$ from the border of the gel as shown in Fig. 5. The distance of $80 \mu \mathrm{m}$ was selected as sufficiently close to the border of the gel to reflect changes in the concentration at the initial stage of the experiment. At the same time, it was sufficiently remote and the results were not influenced by the effects associated with the transition between the gel and the solution. The steep decline in the concentration change after $25 \mathrm{~s}$ is shown in Fig. 5 and relates to the out of range and the simulation data should have a slower decrease.

Such dependence can be achieved using the initial concentration distribution of FMN in the gel with the linear decrease to 0 from the radius centre to the gel border and the following diffusion coefficient $D_{\text {gel }}$

$$
D_{\text {gel }}=\frac{D_{\mathrm{gel}}^{\max }}{1+\exp \left(-\left(t-T_{1}\right) / T_{2}\right)},
$$

where $T_{1}=10 \mathrm{~s}-$ the position of the inflection point, $T_{2}=2.5 \mathrm{~s}-$ time constant, $D_{\text {gel }}^{\max }=0.75 D_{\text {sol }}$ - value of the diffusion coefficient at the end of the swelling [15], $D_{\text {sol }}=4.8^{*} 10^{-10} \mathrm{~m}^{2} / \mathrm{s}$ - diffusion coefficient of FMN in the liquid [16]. As a result, the coincidence of concentrations throughout the experiment was achieved (Fig. 6).

On the basis of this model, the time for beginning of mixing after the introduction of the sample was chosen at the 30th second when most of the FMN escapes the gel.

3.3. Active mixing parameters simulations: The intensity of bioluminescence and the measurement error is largely dependent on the uniform distribution of FMN concentration in the reaction chamber after its escape from the gel. This uniformity can be mathematically expressed with the CV for FMN concentration in the reaction chamber. A lower level of the CV reflects a more uniform distribution of FMN concentration. Sinusoidal and square liquid flow oscillations with 3 and $4 \mu \mathrm{l}$ amplitude at constant and variable frequencies were used to improve mixing.

2D numerical simulations were performed to investigate the distribution of FMN in the reaction chamber after mixing at different flow oscillation frequencies. The basic procedure of FMN concentration simulation in a microfluidic chip was demonstrated previously [17]. The results of the mixing simulations at $1-10 \mathrm{~Hz}$ frequency are shown in Fig. 7. FMN was initially located in the left half of the reaction chamber. When mixing started, the first push at low $1 \mathrm{~Hz}$ frequency moved FMN in the area of immobilised bioluminescent components. Every next push was in the opposite direction according to the signal pattern shown in Fig. 8. A subsequent growth of the oscillation frequency provided a uniform distribution of FMN concentration throughout the reaction chamber by the 3rd second.

It was observed that, at frequencies over $10 \mathrm{~Hz}$, the form of the liquid streams in the chamber changed, resulting in reduced 


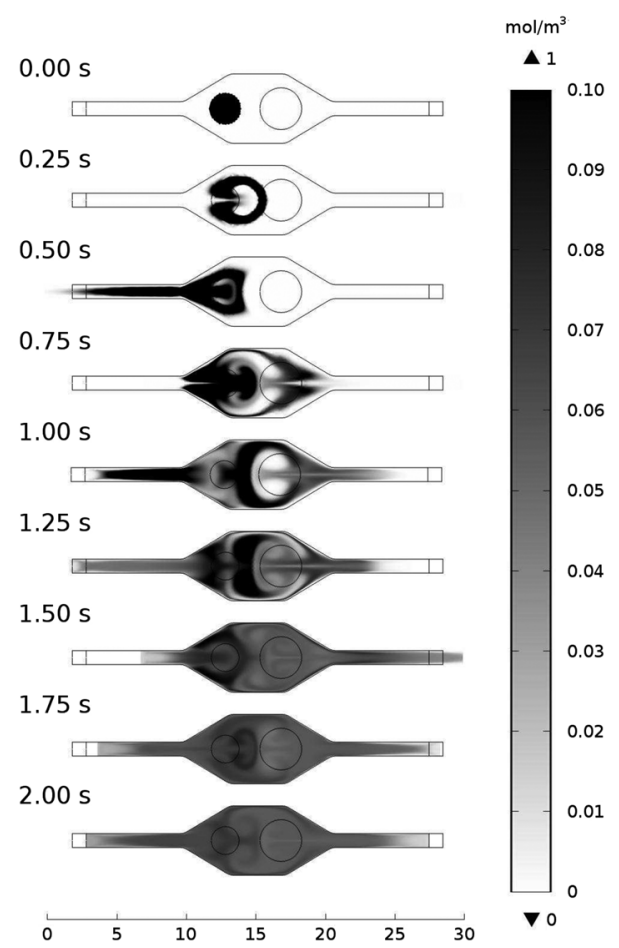

Fig. 7 Simulation of FMN mixing in chip at 1-10 Hz oscillation frequency

mixing efficiency per cycle. Rectangular flow oscillation with a constant frequency showed the least efficient mixing, however, the use of variable frequency provided the same value as the sinusoidal signal as shown in Fig. 9. Thus, the variable frequency of the signal can increase the mixing efficiency in one period of oscillation, since at the same value of the $\mathrm{CV}$ it has the average frequency of $5 \mathrm{~Hz}$. An increase of the oscillation amplitude up to $4 \mu \mathrm{l}$ using this mode allowed to achieve $\mathrm{CV}$ value of FMN concentration in the reaction chamber equal to 0.01 by the 3 rd second.

We used the mixing parameters with a gradual increase of the oscillation frequency from 1 to $14 \mathrm{~Hz}$ for $3 \mathrm{~s}$ as shown in Fig. 8 due to

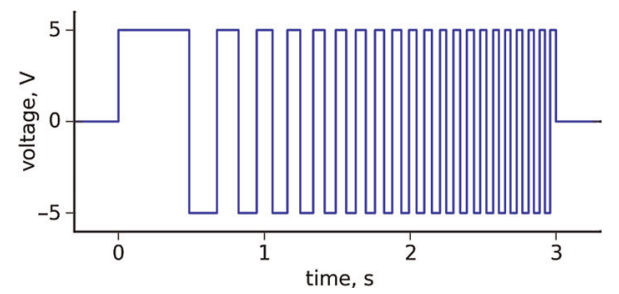

Fig. 8 Mixing pattern for $1-14 \mathrm{~Hz}$ frequency used in the experiment

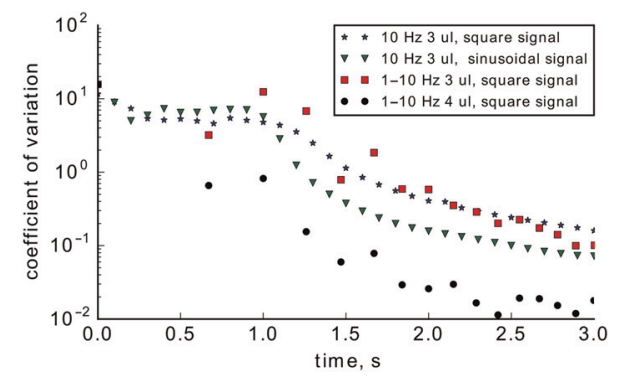

Fig. 9 CV of FMN distribution in the area of enzymes location (Fig. 1) in the reaction chamber of microfluidic chip at the two-dimensional simulation with different signal forms and fluid oscillation amplitude
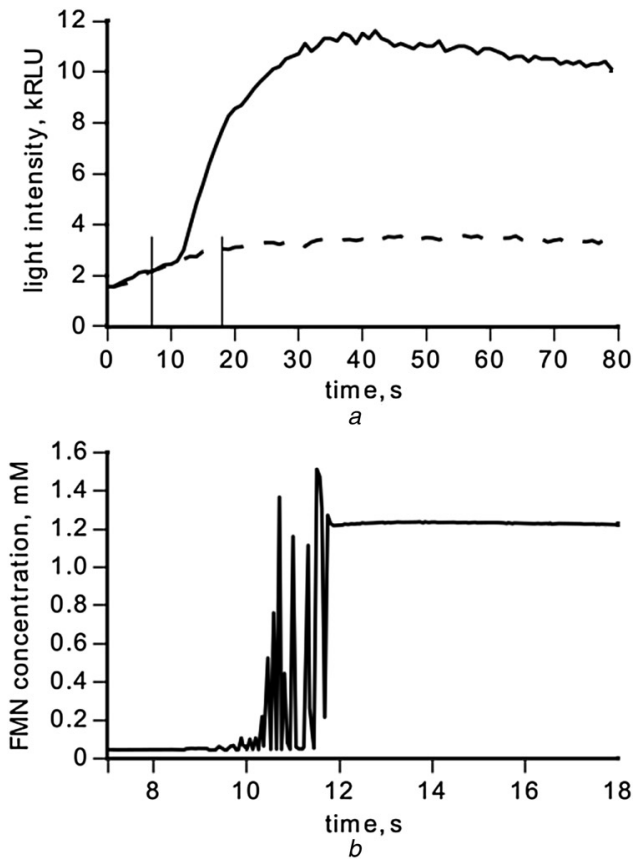

Fig. 10 Experimental verification showed that the intensity of the luminescence signal at the selected mixing parameters increased

$a$ Luminescence kinetics without active mixing (dashed line) and with active mixing (solid line) in a microfluidic chip. Two vertical lines indicate the time interval that was used for the figure below

$b$ FMN concentration in the area of immobilised bioluminescent components when mixing starts at the 10th second of luminescence measurement

the lowest $\mathrm{CV}$ of FMN for the experiments. The rate of frequency change is described by the relationship $y=4,2 x+1$.

3.4. Bioluminescence intensity measurements: The results of the numerical simulation for the chosen reaction chamber geometry, the mixing parameters and FMN gel escape observation were verified experimentally. Time-resolved spectroscopy by HSV palette analysis was used for this purpose.

Experimental verification showed that the intensity of the luminescence signal at the selected mixing parameters increased (Fig. 10a, solid line) from 2000 to 12,000 RLU when applying active mixing compared with passive mixing (Fig. 10a, dashed line). After the beginning of mixing at the 10th second, the FMN concentration distribution became uniform all around the reaction chamber (Fig. 10b) and increased from 0.02 to $1.2 \mathrm{mM}$. Application of active mixing provided the most uniform distribution of FMN concentration in the reaction chamber of the chip and thus reduced the standard deviation to a level of $25 \%$ compared with passive mixing. Further studies showed similar results at constant rectangular frequency of $8 \mathrm{~Hz}$.

The efficiency of the selected mode of mixing may be explained as follows: the velocity profile has a parabolic shape at lower frequencies of fluid oscillation, whereby, FMN at the initial cycles is more evenly distributed throughout the area of the reaction chamber (Fig. 10b). The subsequent increase of frequency and the intensity of pulses resulted in a most efficient mixing.

4. Conclusions: The selected design of the reaction chamber allowed the most efficient use of the limited space on the chip and provided the lowest probability of bubble formation during the introduction of the sample.

Numerical simulation and experimental observation of FMN escape from the gel allowed to predict its concentrations at different times to find the proper time when mixing began. 
The use of the variable frequency of liquid oscillations allowed to achieve the lowest coefficient of the variation of FMN concentration in the reaction chamber.

The results of numerical simulations were verified experimentally. The selected mixing parameters enabled us to provide the reagents escape from the dried gel and to achieve a uniform distribution of FMN in the reaction chamber, which resulted in higher bioluminescent signal maintaining assay reproducibility. The mixing did not affect the kinetics of bioluminescence because the duration of mixing ( $3 \mathrm{~s}$ ) was less than the time required to reach the maximum intensity of luminescence.

The proposed approach for dried reagents mixing may be used with many other microfluidic systems in producing POC devices.

5. Acknowledgment: The research was supported by the grant of the Russian Science Foundation (project no.15-19-10041).

\section{References}

[1] Gubala V., Harris L.F., Ricco A.J., ET AL.: 'Point of care diagnostics: Status and future', Anal. Chem., 2012, 84, (2), pp. 487-515

[2] Jung W., Han J., Choi J., ET AL.: 'Point-of-care testing (POCT) diagnostic systems using microfluidic lab-on-a-chip technologies', Microelectron. Eng., 2015, 132, pp. 46-57

[3] Mark D., Haeberle S., Roth G., ET AL.: 'Microfluidic lab-on-a-chip platforms: requirements, characteristics and applications', Chem. Soc. Rev., 2010, 39, (3), pp. 1153-1182

[4] Evstrapov A.A.: 'Microfluidic chips for biological and medical research', Russ. J. Gen. Chem., 2012, 82, (12), pp. 2132-2145

[5] Roda A., Mirasoli M., Michelini E., ET AL.: 'Progress in chemical luminescence-based biosensors: a critical review', Biosens. Bioelectron., 2016, 76, pp. 164-179
[6] Kratasyuk V.A., Esimbekova E.N.: 'Applications of luminous bacteria enzymes in toxicology', Comb. Chem. High Throughput Screening, 2015, 18, (10), pp. 952-959

[7] Weigl B., Domingo G., LaBarre P., ET AL.: 'Towards nonand minimally instrumented, microfluidics-based diagnostic devices', Lab Chip, 2008, 8, (12), pp. 1999-2014

[8] Lee C.Y., Chang C.L., Wang Y.N., ET AL.: 'Microfluidic mixing: a review', Int. J. Mol. Sci., 2011, 12, (5), pp. 3263-3287

[9] Chin C.D., Linder V., Sia S.K.: 'Commercialization of microfluidic point-of-care diagnostic devices', Lab Chip, 2012, 12, (12), pp. 2118-2134

[10] Gervais L., De Rooij N., Delamarche E.: 'Microfluidic chips for point-of-care immunodiagnostics', Adv. Mater., 2011, 23, (24), pp. H151-H176

[11] Wei S., Vaidya B., Patel A.B., ET AL.: 'Photochemically patterned poly(methyl methacrylate) surfaces used in the fabrication of microanalytical devices.', J. Phys. Chem. B, 2005, 109, (35), pp. $16988-16996$

[12] Shah J.J., Geist J., Locascio L.E., ET AL.: 'Surface modification of poly (methyl methacrylate) for improved adsorption of wall coating polymers for microchip electrophoresis', Electrophoresis, 2006, 27, (19), pp. $3788-3796$

[13] Long T.M., Prakash S., Shannon M.A., ET AL.: 'Watervapor plasmabased surface activation for trichlorosilane modification of PMMA', Langmuir ACS J. surf. colloids, 2006, 22, (9), pp. 4104-4109

[14] Vesel A., Mozetic M.: 'Surface modification and ageing of PMMA polymer by oxygen plasma treatment', Vacuum, 2012, 86, (6), pp. 634-637

[15] Muhr A., Blanshard J.: 'Diffusion in gels', Polymer, 1982, 23, (7), pp. $1012-1026$

[16] Nguyen H.D., Renslow R., Babauta J., ET AL.: 'A voltammetric flavin microelectrode for use in biofilms', Sens. Actuators B Chem., 2012, 161, (1), pp. 929-937

[17] Belousov K.I., Denisov I.A., Lukyanenko K.A., ET AL.: 'Dissolution and mixing of flavin mononucleotide in microfluidic chips for bioassay', J. Phys. Conf. Ser., 2016, 741, (1), p. 012058 